\title{
Electricity generation and pollutants removal of landfill leachate by osmotic microbial fuel cells with different forward osmosis membranes
}

\section{Nan Jiang}

Donghua University

\section{Li Huang}

Donghua University

Manhong Huang ( $\sim$ huangmanhong@dhu.edu.cn )

Teng Cai

East China Normal University

Jialing Song

Donghua University

Shengyang Zheng

Donghua University

Jili Guo

Donghua University

\section{Zhuang Kong}

Donghua University

Liang Chen

Donghua University

\section{Research}

Keywords: Osmotic microbial fuel cells, Landfill leachate, Electricity generation, Microbial community

Posted Date: January 21st, 2021

DOI: https://doi.org/10.21203/rs.3.rs-66054/v3

License: (c) (i) This work is licensed under a Creative Commons Attribution 4.0 International License.

Read Full License

Version of Record: A version of this preprint was published at Sustainable Environment Research on June 25th, 2021. See the published version at https://doi.org/10.1186/s42834-021-00095-7. 


\section{Abstract}

In this study, thin-film composite with embedded polyester screen, cellulose triacetate with a cast nonwoven and cellulose triacetate with embedded polyester screen (CTA-ES) were examined as the intermediate membranes in osmotic microbial fuel cells (OsMFCs). The reactors were fed with actual landfill leachate and the performance was studied in two operation modes: active layer facing draw solution and active layer facing feed solution (AL-FS). The OsMFC with CTA-ES exhibited the best energy generation (maximum power density: $0.44 \mathrm{~W} \mathrm{~m}^{-2}$ ) and pollutant removal efficiency (ammonia nitrogen: $70.12 \pm 0.28 \%$, total nitrogen: $74.04 \pm 0.33 \%$ ) in the AL-FS mode, which could be ascribed to the lowest internal resistance $(236.75 \mathrm{ohm})$ and highest microbial richness. Pseudomonas was the highest proportion of microbial in OsMFCs. The results of this study has demonstrated the potential of OsMFCs for landfill leachate treatment.

\section{Introduction}

Landfill leachate contains a wide range of organics, inorganics, heavy metals and salts. It is generally characterized with a high concentration of ammonia and a low biodegradable organic matter content [1]. Untreated leachate causes serious hazardous impacts to the surrounding environment, and thus requires prompt and efficient action with suitable treatments [2]. At present, Electro-Fenton Process, Sequencing Batch Reactor, Internal Circulation anaerobic reactor and Upflow Anaerobic Sludge Blanket are commonly used to treat landfill leachate [3]. Due to the complex composition of landfill leachate, these treatments alone are not able to achieve complete removal of the diverse organics from leachate, causing secondary pollution and further affecting the environment and human health. Hence, it is necessary to develop new technologies for the effective removal.

Microbial fuel cells (MFCs) is a new method of generating electrical energy through microbially catalyzed organic oxidation [4]. Prior studies have shown that MFCs are capable to remove pollutants from landfill leachate at low energy consumption $[1,5]$. Compared with conventional leachate treatments, MFC can achieve the same or even higher removal performance, while generating energy and saving aeration costs [5]. Wu et al. [6] summarized the effects of different feed modes, cell structures and leachate loading rates on the energy recovery efficiency of MFCs, and identified chemicals and leachate characteristics that could potentially improve bioelectrochemical activity and energy production. These include high concentration of nitrogen and electrical conductivity, which could improve the electrical performance by reducing the internal resistance. By far, research has been focused on using MFCs to recover bioenergy and value-added products from leachate, but MFC has very limited effect on wastewater treatment. Developing a technology that can remove pollutants and generate energy and simultaneously extract clean water from liquid waste stream will be more competitive than existing wastewater treatment technologies. Forward osmosis (FO) can extract clean water from wastewater, but it only concentrates wastewater without degradation of organic pollutants. The combination of FO and MFCs can address their inherent problems and achieve synergetic benefits. 
Zhang et al. [7] first used FO membrane as a separator to construct a new type of MFCs, and found that osmotic microbial fuel cell (OsMFC) had a higher electricity production than conventional MFCs under sequential batch and continuous operation. Werner et al. [8] constructed an air cathode microbial osmotic fuel cell and observed low internal resistance and high power density. In order to improve the treatment efficiency, Zhang and He [9] coupled an OsMFC with a microbial desalination cell (MDC), and the coupled system significantly improved the desalination efficiency as a combined result of the dilution in the OsMFC and the bioelectrochemical desalination in the MDC. Zhu et al. [10] studied the electrochemical properties of different MFCs with FO membrane, anion exchange membrane and cation exchange membrane as separators. It was found that the FO-MFC has lower internal resistance and a higher output voltage, probably due to higher salt concentration and higher proton flux. To comprehend the influence of membrane materials on OsMFC performance, Yang et al. [11] compared the performance of OsMFCs with FO membranes consist of polyamide (PA), cellulose triacetate with a cast nonwoven (CTA-NW), and cellulose triacetate with embedded polyester screen (CTA-ES). The results demonstrated that the electricity generation and water flux of CTA-ES in OsMFC were higher than CTA-NW and PA. Wu et al. [12] had observed effects of power generation on reverse solute flux (RSF) of ten inorganic-based draw solution (DS) in OsMFCs. $\mathrm{Na}^{+}$-based and $\mathrm{K}^{+}$-based DS were considered to be most suitable DS for OsMFC owing to stable power generation and water flux, as well as significant RSF reduction. These studies have added a growing body of knowledge of the fundamentals of OsMFC. However, the applications of OsMFC in leachate treatment is still lacking.

In this work, the effects of three different FO membranes (thin-film composite with embedded polyester screen (TFC-ES), CTA-NW, and CTA-ES) on OsMFC electricity generation and actual landfill leachate treatment were studied. The reactors were run in two modes: active layer facing feed solution (AL-FS) and active layer facing draw solution (AL-DS). The biomass on OsMFC electrode/membrane surface and in anolyte/catholyte were sampled, and the microbial communities were characterized using 16S rRNA amplicon sequencing. The particular aims of this research were to: (1) demonstrate feasibility of using OsMFC to treat actual landfill leachate; (2) investigate the OsMFCs performance affected by membrane material and membrane orientation; (3) analyze the microbial community at the different locations in the OsMFCs and identify the dominant taxa. This work explored the effects of different FO membrane materials on the power generation and water flux of OsMFCs, which made a contribution to improving the capacity of OsMFCs to treat landfill leachate and provided a reference for future researchers.

\section{Materials And Methods}

\subsection{OsMFC set-up}

The OsMFCs were made of plexiglass, and the effective volume of the anode and cathode chambers was $125 \mathrm{~mL}$. The two chambers were separated by FO membranes (diameter $0.35 \mathrm{~m}$, effective working area is $9.6 \times 10^{-4} \mathrm{~m}^{2}$ ). The anode and cathode electrodes were made of carbon felt (Physicochemical (Hong Kong) Ltd, China), and the projected area of both electrodes is $8 \mathrm{~cm}^{2}(1 \times 8 \mathrm{~cm})$. In order to improve electricity production, the cathode carbon felt was heated at $600^{\circ} \mathrm{C}$ for $4 \mathrm{~h}$ before installation. Titanium 
wire was applied to link the electrodes and the resistance was $200 \Omega$. The cathode was externally connected to a $1000 \mathrm{~mL}$ beaker for catholyte recirculation with a peristaltic pump $\left(60 \mathrm{~mL} \mathrm{~min}^{-1}\right)$ to maintain the dissolved oxygen concentration. The structure of the OsMFC is shown in Fig. S1.

Anode chambers were inoculated with microorganisms from a MFC that had been running steadily for two years. FO membranes used in this experiment are three commercial membranes of TFC-ES, CTA-NW and CTA-ES (HTI AQP, USA). After enrichment, the anolyte (feed solution) was changed to landfill leachate from a landfill in Songjiang, Shanghai. The characteristics of leachate employed are given in Table 1. $\mathrm{NaCl}$ solution $\left(1 \mathrm{~mol} \mathrm{~L}^{-1}\right)$ was chosen as the catholyte (draw solution). The OsMFCs were operated under a sequential batch mode. Samples were collected as soon as the cycles ends. Experiments were performed in a climatic cabinet (Shanghai Bielang Instrument Co., LTD) at $36 \pm 3^{\circ} \mathrm{C}$.

Table 1 Water quality indicators of landfill leachate.

\begin{tabular}{ccc}
\hline Parameter & Unit & Conc. \\
\hline $\mathrm{pH}$ & - & $8.35 \pm 0.03$ \\
Conductivity & $\mathrm{mS} \mathrm{cm}^{-1}$ & $25.40 \pm 0.20$ \\
$\mathrm{SS}$ & $\mathrm{mg} \mathrm{L}^{-1}$ & $896.00 \pm 4.00$ \\
$\mathrm{COD}$ & $\mathrm{mg} \mathrm{L}^{-1}$ & $2638.20 \pm 33.60$ \\
$\mathrm{TOC}$ & $\mathrm{mg} \mathrm{L}^{-1}$ & $716.00 \pm 2.50$ \\
$\mathrm{BOD}_{5}$ & $\mathrm{mg} \mathrm{L}^{-1}$ & $790.00 \pm 5.00$ \\
$\mathrm{NH}_{4}^{+}-\mathrm{N}$ & $\mathrm{mg} \mathrm{L}^{-1}$ & $1666.05 \pm 2.50$ \\
$\mathrm{TN}^{-}$ & $\mathrm{mg} \mathrm{L}^{-1}$ & $2015.90 \pm 6.44$ \\
$\mathrm{TP}$ & $\mathrm{mg} \mathrm{L}^{-1}$ & $3.27 \pm 0.04$ \\
\hline
\end{tabular}

SS: Suspended solids; COD: Chemical oxygen demand; TOC: Total organic carbon; $\mathrm{BOD}_{5}$ : Biochemical oxygen demand; $\mathrm{NH}^{+}{ }^{+} \mathrm{N}$ : Ammonia nitrogen; $\mathrm{TN}$ : Total nitrogen; TP: Total phosphorus.

\subsection{System performance evaluation}

The voltages of the OsMFCs were registered every 2 min via CTA2001A, (Wuhan LAND Electronic Co.Ltd). Both open circuit voltage and operating voltage were collected. Current was calculated according to the Ohm's law $(I=U / R$, where $U(\mathrm{~V})$ is operating voltage, and $R(\Omega)$ is external resistance) and normalized to anode electrode area $\left(I_{A}=I / A\right.$, where $I(\mathrm{~A})$ is current, and $A\left(\mathrm{~m}^{2}\right)$ is projected area of anode electrode) to obtain current density. Power output was also calculated using the Ohm's law $(P=U I / A)$. Polarization curve and the power density curve were measured with the steady-state discharge method. Before testing, OsMFCs were stabilized in open circuit for 12 hours. The resistance was the decreased consecutively 
between $10000 \Omega$ and $20 \Omega$ with a rheostat (Shanghai Dongmao Electronic Technology Co., LTD), and the voltages under different resistances were recorded. Total organic carbon (TOC) was gauged by a TOC detector (Vcph, Shimadzu, Japan). Ammonia nitrogen $\left(\mathrm{NH}^{+}-\mathrm{N}\right)$ was measured by Nessler's reagent spectrophotometry. Total phosphorus (TP) was measured using Molybdenum-antimony spectrophotometry. Total nitrogen (TN) was measured by spectrophotometry method (HJ-636-2012, China).

The mass change of the draw solution was measured with an electronic balance (ME104/02, Shimadzu, Japan) to measure the water flux $J w\left(L /\left(\mathrm{m}^{2} \cdot \mathrm{h}\right)\right)$ :

$$
J w=\frac{\Delta m}{A_{m} \times \Delta t}
$$

Where $\Delta t(\mathrm{~h})$ is the operation time, $\Delta m(\mathrm{~g})$ is increased weight of DS during operation time, $A_{m}\left(\mathrm{~m}^{2}\right)$ is actual membrane surface area $\left(9.616 \times 10^{-4} \mathrm{~m}^{2}\right)$.

The reverse salt flux $J s\left(\mathrm{~g} / \mathrm{m}^{2} \cdot \mathrm{h}\right)$ was figured as below $\mathbb{Z}$

$$
J_{\mathrm{S}}=\frac{V_{t} \times C_{t}-V_{0} \times C_{0}}{A_{m} \times \Delta t}
$$

Where $V_{0}(\mathrm{~L})$ is original volume of feed solution, $V_{t}(\mathrm{~L})$ is final volume of feed solution, $C_{0}(\mathrm{mg} / \mathrm{L})$ is original salt concentration of feed solution, $C_{t}(\mathrm{mg} / \mathrm{L})$ is the final salt concentration of feed solution, $\Delta t$ (h) is operation time, $A_{m}\left(\mathrm{~m}^{2}\right)$ is effective membrane surface area $\left(9.616 \times 10^{-4} \mathrm{~m}^{2}\right)$.

\subsection{Microbial analysis}

Samples were collected from anode carbon felt, membranes, anolyte, and catholyte in the OsMFCs equipped with three different FO membranes (CTA-ES, CTA-NW, and TFC-ES). Then, according to the manufacturer's (Shenggong Biotechnology Co., Ltd., Shanghai, China) instructions, DNA was extracted from samples using the Soil DNA kit. The first round of polymerase chain reaction amplification was performed using the primers targeting the V3/V4 region of the bacterial $16 \mathrm{~S}$ rRNA genes: 341F (CCCTACACGACGCTCTTCCGATCTG (barcode) CCTACGGGNGGCWGCAG) and 805R (GACTGGAGTTCCTTGGVACCCGAGAATTCCAGACTACHVGGGTATCTAATCC), followed by a second round of amplification with the same primer pairs. After amplification, gel electrophoresis was performed to detect the amplified products. A magnetic beads (Agencourt AMPure XP) with a volume ratio of 0.6 to the sample was selected for purification and recovery of DNA fragments longer than $400 \mathrm{bp}$. The Qubit 2.0 
DNA analysis kit was used to accurately quantify the recovered DNA [13]. The amplicon was pooled at 10 ng DNA per sample, resulting in a final sequencing concentration of 20 pmol.

The ribosomal database project classifier is used to classify the sequences, classify each sample and each species unit, perform sequence abundance calculation, and construct a sequence abundance matrix of sample and species classification units.

The software used for Alpha diversity analysis is mothur, and its indicators include Shannon Index, Abundance-based Coverage Estimator (ACE) Index, Chao1 Index, Coverage, Simpson, etc.

\section{Results And Discussion}

\subsection{OsMFCs performance with different FO membranes and operation modes}

\subsubsection{Power generation}

Fig. 1A shows the electricity production of the OsMFCs with three different membranes under AL-FS mode. The voltage of CTA-ES-OsMFC remained high throughout the experiment, while that of the CTANW-OsMFC was high at the beginning, dropped rapidly in a first $2000 \mathrm{~min}$ of operation, and remained relatively stable. In comparison, the voltage of the CTA-NW-OsMFC was the lowest. When the reactors reached steady state, the output voltages of TFC-ES-OsMFC, CTA-NW-OsMFC, and CTA-ES-OsMFC were $108.8,106.9$, and $153.5 \mathrm{mV}$, respectively. The high voltage output with the CTA-ES membrane might be due to the high water flux in that OsMFC, which accelerated ion transfer and consequently reduce internal resistance. Although the water flux (Fig. 3) with the TFC-ES membrane was also high, the growth of the anode bacteria might be limited due to the higher oxygen diffusion $[11,13]$. It can be seen from Fig. 1B the electricity production of the three OsMFCs under the AL-DS mode was $148.7,87.4$, and $79.8 \mathrm{mV}$, for CTA-ES-OsMFC, CTA-NW-OsMFC, and TFC-ES-OsMFC, respectively. It was worth noting that the voltages of the CTA-NW-OsMFC and TFC-ES-OsMFC under the AL-DS mode were 18.2 and $26.7 \%$ lower than those under the AL-FS mode, respectively, which might be due to more serious membrane fouling and faster drop of water flux on AL-DS mode [14]. When comparing with TFC-ES-OsMFC, CTA-NW-OsMFC showed lower voltage in AL-FS mode but higher voltage in AL-DS mode. In the AL-FS mode, the voltage of CTANW-OsMFC was lower than that of TFC-ES-OsMFC owing to CTA-NW had a larger structural parameter $(S)$ value. It was known that the larger the $S$ value of the membrane was, the larger the mass transfer resistance would be ( $S$ value: CTA-NW (1.38) > TFC-ES $(0.205 \mathrm{~mm})$ ) [11]. Even though TFC-ES exhibited a lower mass transfer resistance than CTA-NW, it displayed a lower power generation of the OsMFC test in AL-DS mode. This result could be attributed to the higher oxygen diffusion through TFC-ES. Diffusion of oxygen from the cathode chamber would limit constrain viability of anode bacteria, thereby reducing the output voltage $[11,12]$.

The polarization curves and power density curves at the initial operation stage were shown in Fig. 2(A-B). Under the AL-FS mode, the open circuit voltages of CTA-ES-OsMFC, CTA-NW-OsMFC, and TFC-ES-OsMFC were $614.6,545.7$, and $551.8 \mathrm{mV}$, respectively, which were not significantly different from the open circuit 
voltage under AL-DS mode (590.7, 548.8, 551.2 mV). According to the slope of polarization curve [10], the internal resistance of CTA-ES-OSMFC, CTA-NW-OsMFC, and TFC-ES-OsMFC under AL-FS mode were $236.75,392.37$, and $307.62 \Omega$, respectively, which were $28.26,22.78$, and $20.23 \%$ lower than that under AL-DS mode. As a critical parameter in OsMFCs internal resistance is directly linked to power density. The maximum power density of the OsMFCs exhibited a trend in the following order: FS-CTA-ES-OsMFC (0.44) $>$ FS-TFC-ES-OsMFC (0.37) > DS-CTA-ES-OsMFC (0.33) > DS-TFC-ES-OsMFC (0.30) > FS-CTA-NW-OsMFC $(0.28)>$ DS-CTA-NW-OsMFC $\left(0.24 \mathrm{~W} \mathrm{~m}^{-2}\right)$. As an important parameter of FO membranes, lower $S$ value mean a reduction in internal concentration polarization (ICP) [15]. Thus, the lower the $S$ value, the better the permeability of FO membrane [16]. The $S$ values of CTA-NW, CAT-ES and TFC-ES membranes were $1.38,0.334$, and $0.205 \mathrm{~mm}$, respectively [11]. The reason why CTA-NW-OsMFC generated the lowest current and maximum power density might be that the relatively large $S$ value led to the increase in mass transfer resistance, which weakened the power generation capacity of the system [11]. To achieve high power generation, FO membranes used in OsMFCs should not only maintain a good mass transfer rate, but also minimize oxygen diffusion, as the intruded oxygen can serve as an alternative electron acceptor to the anode bacteria and short-circuit the system.

\subsubsection{Reverse salt flux and water flux}

Reverse salt flux and water flux of reactor were also important indicators to evaluate the OsMFC performance. Fig. 3 shows the reverse salt flux and water flux of three OsMFCs. Under the AL-FS mode, the order of reverse salt flux and water flux of the membranes were: CTA-ES (3.68) > TFC-ES (3.37) > CTANW (3.01 gMH), and CTA-ES (0.98) > TFC-ES (0.79) > CTA-NW (0.56 LMH), respectively. The ratios between water flux and reverse salt flux were thus $0.27,0.18$, and $0.23 \mathrm{~L} \mathrm{~g}^{-1}$, respectively. Under the AL-DS mode, the order of reverse salt flux and water flux of membranes were: CTA-ES (4.28) > TFC-ES (4.28) > CTA-NW (4.25 gMH), and CTA-ES (1.02) > TFC-ES (0.84) > CTA-NW (0.71 LMH). The ratios between water flux and reverse salt flux were $0.24,0.17$, and $0.20 \mathrm{~L} \mathrm{~g}^{-1}$, respectively. The reverse salt flux and water flux under the AL-FS mode were both smaller than that under the AL-DS mode, and CTA-ES in those two modes showed better performance than the other two membranes, which was consistent with the previous results (Fig. 1 and 2). Under AL-FS mode, draw side was diluted with the formation of ICP, then reduced osmotic pressure difference in two sides of membrane. In the meantime, draw solute entered the membrane through the porous support layer, increasing the resistance over the membrane, which might be the reason for this difference [17]. However, microorganisms and macromolecular organic substances in the anolyte might block porous support layer under AL-DS mode, thus increasing membrane resistance. Therefore, the difference of water flux on AL-DS and AL-FS mode was not particularly significant.

\subsubsection{Pollutant removal performance}

In Fig. 4, the removal efficiencies of TOC, total nitrogen and TP by three OsMFCs under the two modes were very similar, with no obvious difference. All OsMFCs had a removal efficiency of more than $70 \%$ for TOC and total nitrogen, and more than $85 \%$ for TP. Once active layer of membrane faced anolyte (AL-FS mode), some of microorganisms in the anolyte deposited on the membrane surface, causing membrane 
clogging and thereby affecting the contaminant removal [18]. In AL-DS mode, the effective mass transfer driving force was large, and the pollutants formed a higher concentration gradient in support layer under concentrated concentration polarization, so that more pollutants reached the cathode [19]. Therefore, the removal efficiencies of the OsMFCs under the two modes were similar in this experiment. However, the removal of ammonia nitrogen was not as efficient. Among the OsMFCs, DS-CTA-NW-OsMFC had the lowest removal efficiency for ammonia nitrogen, which was only $52.43 \pm 0.41 \%$. In contrast, the OsMFCs under the AL-FS mode showed higher removal efficiencies for ammonia nitrogen than under the AL-DS mode. FS-CTA-ES-OsMFC achieved the highest ammonia nitrogen removal of $70.12 \pm 0.28 \%$. It has been reported that, to maintain charge balance, $\mathrm{NH}^{+}{ }_{-}^{+} \mathrm{N}$ in the anolyte migrates into the catholyte when $\mathrm{Na}^{+}$ diffused into the anolyte [20]. In OsMFCs, the FO membrane surface carried more negative charge, which led to an increase in the bidirectional diffusion of $\mathrm{NH}_{4}{ }^{+}$and $\mathrm{Na}^{+}$[20]. A previous study has reported that ammonium ion movement from the anode to the cathode promoted by water flux [20]. From Fig 4, the FO test of CTA-ES membrane showed the highest water flux in both AL-FS and AL-DS modes. Therefore, OsMFCs with CTA-ES membrane had the highest ammonia nitrogen removal efficiency. In AL-DS mode, although the membrane water flux was high, it would produce severe ICP and membrane fouling, thus reducing the removal effect of pollutants [19]. Overall, the removal of various pollutants in landfill leachate was satisfactory compared with that in the published literature (Table S1), which proved that OsMFCs is a promising technology for landfill leachate treatment.

\subsection{Microbial community structure on different FO membranes}

\subsubsection{Microbial diversity}

The abundance and diversity of bacterial community could be revealed through diversity analysis. Table 2 listed the Alpha diversity indices of the microbial communities in the three OsMFCs. The ACE and Chao1 were indices that estimate number of operational taxonomic units (OTUs) in a community and were commonly used to estimate the total number of species. Table 2 showed that both the ACE index and the Chao 1 index follow the same order: CTA-ES-OsMFC > CTA-NW-OsMFC > TFC-ES-OsMFC. The Shannon and Simpson index are used to estimate the microbial diversity in a sample: a high diversity is reflected by a high Shannon index and a low Simpson index. As shown in Table 2, the ranking of the Shannon index was CTA-NW-OsMFC (5.09) > CTA-ES-OsMFC (4.73) > TFC-ES-OsMFC (4.35). Correspondingly, the order of the Simpson index was CTA-NW-OsMFC $(0.02)<$ CTA-ES-OsMFC $(0.03)<$ TFC-ES-OsMFC (0.05). Both indices indicated a highly diverse microbial community on the CTA-NW membrane. The diversity analysis results are reliable as indicated by the high coverage index of 0.99 for the three OsMFCs. The rarefaction curve (Fig. S2) also indicates that CTA-NW-OsMFC had the highest number of microbial species, while TFC-ES-OsMFC had the lowest number of species.

Table 2 Species diversity of microbial communities on different membranes 


\begin{tabular}{cccccccc}
\hline Sample-ID & Seq-num & OTU & ACE & Chao1 & Shannon & Simpson & Coverage \\
\hline CTA-NW-OsMFC & 62582 & 1526 & 1905.85 & 1822.93 & 5.09 & 0.02 & 0.99 \\
CTA-ES-OsMFC & 75625 & 1516 & 1909.36 & 1865.47 & 4.73 & 0.03 & 0.99 \\
\hline TFC-ES-OsMFC & 47491 & 1126 & 1527.06 & 1473.72 & 4.35 & 0.05 & 0.99 \\
\hline
\end{tabular}

\subsubsection{Microbial community composition}

At the genus level, the community structure on the different membranes was similar, but the abundance of the genus was different (Fig. 5A). Genera belonging to Proteobacteria (Pseudomonas, Thiopseudomonas, Marinobacterium, Arcobacter, Sulfurospirillum, Rhodopseudomonas, Desulfobulbus, Pusillimonas, Advenella), Firmicutes (Saccharofermentans and Tissierella), Bacteroidetes (Proteiniphilum), and Synergistetes (Aminobacterium) were the dominant taxa on the membrane surface.

Proteobacteria is mostly anaerobic or facultative anaerobic gram-negative bacteria. It was widely distributed on the membrane surface, and the percentages in CTA-ES-OsMFC, CTA-NW-OsMFC, and TFCES-OsMFC were $40.11,47.21$, and $68.11 \%$, respectively. Many studies had shown that Proteobacteria exists extensively in MFC anodes [21, 22], thus key genera are discussed in details below.

Pseudomonas is a heterotrophic bacterium under the $\gamma$-proteobacteria class, which uses organic matter as a energy source for anaerobic respiration [23]. Pseudomonas accounted for $6,13.56$, and $20.55 \%$ in the CTA-NW-OsMFC, CTA-ES-OsMFC, and TFC-ES-OsMFC, respectively, and thus was speculated to be the major electroactive bacteria in the OsMFCs. It could also be seen from Fig. S3 that Pseudomonas was most abundant on the three membranes. Pham et al. [23] found that Pseudomonas produced phenazine compounds for electron shuttling and improved MFC performance in mixed cultures. The abundance of Marinobacterium in the TFC-ES-OsMFC (4.42\%) was relatively higher than in the CTA-NW-OsMFC (1.14\%) and CTA-ES-OsMFC (2.04\%). Marinobacterium has been reported to be halophiles [24]. Because the catholyte was $1 \mathrm{~mol} \mathrm{~L}^{-1} \mathrm{NaCl}$, the forward osmosis would cause reversely transported salts. The landfill leachate also contained a certain salinity, resulting in high salinity of the anolyte. Arcobacter belongs to the $\varepsilon$-proteobacteria class whose bioelectrochemical activity has been reported [25]. The percentage of Arcobacter in CTA-ES-OsMFC (9.54\%) was higher than in CTA-NW-OsMFC (6.01\%) and TFC-ES-OsMFC (0.35\%). This might be one of the reasons why CTA-ES-OsMFC had a better power generation effect (Fig. 2). Fedorovich et al. [26] found that Arcobacter butzleri ED-1 was an electrogenic microorganism that could efficiently use acetate as a carbon source. Due to the presence of Saccharofermentans in OsMFCs, the organic matter is degraded into acetic acid, which leads to better growth of Arcobacter.

Rhodopseudomonas belongs to the a-proteobacteria class, is a Gram-negative bacterium $\Downarrow$ which secretes a phenolic electron mediator with weak electrical energy and uses various carbon as an energy source to generate electricity [26]. Rhodopseudomonas in CTA-NW-OsMFC, CTA-ES-OsMFC, and TFC-ES-OsMFC were $3.8,4.43$, and $2.86 \%$, respectively. Thiopseudomonas as a denitrifying bacteria [27] showed a percentage of $1.58,1.57$, and $10.05 \%$ in CTA-NW-OsMFC, CTA-ES-OsMFC, and TFC-ES-OsMFC, respectively. 
Firmicutes have been reported to utilize complex organic matter to produce electricity [28]. The abundance of Firmicutes in the CTA-ES-OsMFC, CTA-NW-OsMFC, and TFC-ES-OsMFC were 28.85, 24.23, and $11.80 \%$, respectively, probably due to the presence of complex high organic in landfill leachate. In this phylum, Saccharofermentans are known to perform hydrolysis, fermentation, and acetogenesis [29]. Its abundance in CTA-NW-OsMFC, CTA-ES-OsMFC, and TFC-ES-OsMFC were 3.34, 3.58, and 2.24\%, respectively.

Pseudomonas, Arcobacter and Rhodopseudomonas are commonly found in bioelectrochemical systems and are speculated to be the main electrogenic bacteria in the OsMFCs [30]. However, some wellknown electroactive microorganisms (e.g., Anaeromusa, Dechlormonas, Geobacter, and etc.) were not found in this study, probably because the salt in the catholyte passed through the membranes and caused high anode salinity. Anaeromusa and Geobacter microbes existed extensively in substrate where lactate and citrate were carbon sources [31,32]. Dechlormonas was more active in domestic wastewater [32]. In addition, landfill leachate as an anode substrate has a complex composition and high toxicity that may be inhibitory to those microorganisms.

\subsection{Microbial community structure at different OsMFCs sites}

\subsubsection{Microbial diversity}

It can be seen from Table 3 that the ACE index and Chao 1 index are the highest in the anolyte (1928.77, 1875.12) compared to the TFC-ES membrane $(1527.06,1473.72)$, anode carbon felt $(1579.99,1513.01)$, and catholyte $(222.52,208.63)$. Similarly , the anolyte sample showed the highest Shannon index and the lowest Simpson index, suggesting that the anolyte community had high richness and diversity [33]. The same result could also be proven in the rarefaction curves (Fig. S4). As shown in Table 3, the coverage index of the four samples indicated that the sequencing results has captured the dominant taxa in the communties.

Table 3 Species diversity of microbial communities on different OsMFCs sites

\begin{tabular}{cccccccc}
\hline Sample-ID & Seq-num & OTU & ACE & Chao1 & Shannon & Simpson & Coverage \\
\hline Membrane & 47491 & 1126 & 1527.06 & 1473.72 & 4.35 & 0.05 & 0.99 \\
Electrode & 63407 & 1241 & 1579.99 & 1513.01 & 4.74 & 0.03 & 0.99 \\
Anolyte & 64396 & 1536 & 1928.77 & 1875.12 & 5.09 & 0.02 & 0.99 \\
Catholyte & 64566 & 200 & 222.52 & 208.63 & 1.69 & 0.28 & 1.00 \\
\hline
\end{tabular}

\subsubsection{Microbial community composition}

As shown in Fig. 5B, there were some differences in community structure for four samples of TFC-ESOsMFCs. Aliidiomarina, Halomonas, Pseudomonas, Rhodopseudomonas, Methylophaga, Thiopseudomonas, Marinobacterium, Desulfuromonas (these genus belong to Proteobacteria phylum), 
Saccharofermentans, Tissierella, Sporanaerobacter (belong to the Firmicutes phylum) were the dominant species of four samples.

The Proteobacteria phylum was gain dominant in the communities, and the abundance of Proteobacteria on the TFC-ES membrane, anode carbon felt, in the anolyte, and catholyte were $68.11,36.02,36.52$, and $97.62 \%$, respectively. Aliidiomarina was found to be significantly enriched in the catholyte with an abundance of $40.59 \%$ but not at other locations. This result was the same as shown in Fig. S5. Halomonas, a rod-shaped gram-negative bacteria that can survive in saline environments [34], was also abundant with $31.16 \%$ only in the catholyte. Methylophaga as an aerobic Gram-negative moderate halophilic genus that utilizes a single carbon sugar in the ribulose monophosphate pathway as a carbon source showed a high abundance of $14.97 \%$ in the catholyte [35]. The predominance of those taxa was correlated with the higher salinity in catholyte.

The abundance of Pseudomonas detected on the TFC-ES membrane, anode carbon felt, in the anolyte, and catholyte samples were $20.55,1.87,7.88$, and $0.58 \%$. A small amount of Pseudomonas was also found in the catholyte, probably because a part of the microorganisms permeated into the catholyte during the operation. The abundance of Rhodopseudomonas genus on the TFC-ES membrane, anode carbon felt, in the anolyte were $2.86,13.35$, and $6.64 \%$, respectively. The high abundance of Rhodopseudomonas on the carbon felt reflected its electroactive nature. Desulfuromonas can grow through oxidizing acetate and reducing elemental sulfur [36]. The abundance of this taxa was as high as $5.11 \%$ on the carbon but low in membrane $(0.11)$ and anolyte $(0.68 \%)$ and not detected in catholyte. Thiopseudomonas was observed relatively abundant on the carbon felt $(1.32 \%)$ and in the anolyte (3.04\%).

\section{Conclusions}

The CTA-ES-OsMFC showed the highest electricity production and most efficient pollutant removal under both operation modes. Under the AL-FS mode, CTA-ES-OsMFC achieved the highest power output of 0.44 $\mathrm{W} \mathrm{m} \mathrm{m}^{-2}$ with $76.63 \pm 0.28 \% \mathrm{TOC}$ removal, higher than the $0.33 \mathrm{~W} \mathrm{~m}^{-2}$ and $74.06 \pm 0.28 \%$ under the AL-DS mode. Sequencing analyses revealed that the CTA-ES-OsMFC had the highest microbial enrichment on the membrane, which might explain the satisfactory performance of the CTA-ES-OsMFC. Pseudomonas was the most abundant taxa on the membranes, and Rhodopseudomonas was the most abundant taxa on the carbon felt, both of which were likely to the the key electrogenic bacteria in the OsMFCs.

\section{Declarations}

\section{Availability of data and materials}

All data generated or analysed during this study are included in this published article and its supplementary information files. 
The authors declare that they have no competing interests in this section.

\section{Funding}

This work was supported by the National Key Research Development Program of China (2019YFC0408304), the Fundamental Research Funds for the Central Universities (No. 2232020G-04).

\section{Authors' contributions}

Nan Jiang and Li Huang have conducted the experimental studies on osmotic microbial fuel cells for the treatment of Landfill leachate. Teng Cai and Jialing Song have supervised towards the experimental work on power generation and performance studies. Manhong Huang and Liang Chen have expressed their suggestions and ideas on the chemical analysis and characteristics of the OsMFC. Shengyang Zheng, Jili Guo and Zhuang Kong helped revise the manuscript. All authors read and approved the final manuscript.

\section{Acknowledgments}

The authors gratefully acknowledge the financial support given by the National Key Research Development Program of China (2019YFC0408304) and the Fundamental Research Funds for the Central Universities (No. 2232020G-04).

\section{References}

1. Nguyen $\mathrm{HTH}$, Min B. Leachate treatment and electricity generation using an algae-cathode microbial fuel cell with continuous flow through the chambers in series. Sci Total Environ. 2020;723:138054.

2. Tugtas AE, Cavdar P, Calli B. Bio-electrochemical post-treatment of anaerobically treated landfill leachate. Bioresour Technol. 2013;128:266-72.

3. Aftab B, Ok YS, Cho J, Hur J. Targeted removal of organic foulants in landfill leachate in forward osmosis system integrated with biochar/activated carbon treatment. Water Res. 2019;160:217-27.

4. Cai T, Meng LJ, Chen G, Xi Y, Jiang N, Song JL, et al. Application of advanced anodes in microbial fuel cells for power generation: A review. Chemosphere. 2020;248:125985.

5. Greenman J, Gálvez A, Giusti L, leropoulos I. Electricity from landfill leachate using microbial fuel cells: Comparison with a biological aerated filter. Enzyme Microb Technol. 2009;44(2):112-9.

6. Wu D, Wang T, Huang XH, Dolfing J, Xie B. Perspective of harnessing energy from landfill leachate via microbial fuel cells: novel biofuels and electrogenic physiologies. Appl Microbiol Biotechnol. 2015;99(19):7827-36.

7. Zhang F, Brastad KS, He Z. Integrating forward osmosis into microbial fuel cells for wastewater treatment, water extraction and bioelectricity generation. Environ Sci Technol. 2011;45(15):6690-6.

8. Werner CM, Logan BE, Saikaly PE, Amy GL. Wastewater treatment, energy recovery and desalination using a forward osmosis membrane in an air-cathode microbial osmotic fuel cell. J Membr Sci. 2013;428:116-22. 
9. Zhang B, He Z. Improving water desalination by hydraulically coupling an osmotic microbial fuel cell with a microbial desalination cell. J Membr Sci. 2013;441:18-24.

10. Zhu XZ, Zhang F, Li WW, Liu HQ, Wang YK, Huang MS. Forward osmosis membrane favors an improved proton flux and electricity generation in microbial fuel cells. Desalin. 2015;372:26-31.

11. Yang E, Chae KJ, Kim IS. Comparison of different semipermeable membranes for power generation and water flux in osmotic microbial fuel cells. J Chem Technol Biotechnol. 2016;91(8):2305-12.

12. Wu SM, Qian GR, He Z. Examination of inorganic-based draw solutes and mitigation of their reverse solute flux in osmotic microbial fuel cells. J Chem Technol Biotechnol. 2019;94(7):2107-14.

13. Huang L, Li XC, Cai T, Huang MH. Electrochemical performance and community structure in three microbial fuel cells treating landfill leachate. Process Saf Environ Prot. 2018;113:378-87.

14. Honda R, Rukapan W, Komura H, Teraoka Y, Noguchi M, Hoek EMV. Effects of membrane orientation on fouling characteristics of forward osmosis membrane in concentration of microalgae culture. Bioresour Technol. 2015;197:429-33.

15. Kim B, Gwak G, Hong S. Review on methodology for determining forward osmosis (FO) membrane characteristics: Water permeability (A), solute permeability (B), and structural parameter (S). Desalin. 2017;422:5-16.

16. Tiraferri A, Yip NY, Straub AP, Castrillon SRV, Elimelech M. A method for the simultaneous determination of transport and structural parameters of forward osmosis membranes. J Membr Sci. 2013;444:523-38.

17. Liu XJ, Wu JL, Liu C, Wang JL. Removal of cobalt ions from aqueous solution by forward osmosis. Sep Purif Technol. 2017;177:8-20.

18. Yang E, Chae KJ, Choi MJ, He Z, Kim IS. Critical review of bioelectrochemical systems integrated with membrane-based technologies for desalination, energy self-sufficiency, and high-efficiency water and wastewater treatment. Desalin. 2019;452:40-67.

19. Wang Y, Wicaksana F, Tang CY, Fane AG. Direct Microscopic Observation of Forward Osmosis Membrane Fouling. Environ Sci Technol. 2010;44(18):7102-9.

20. Qin MH, Hynes EA, Abu-Reesh IM, He Z. Ammonium removal from synthetic wastewater promoted by current generation and water flux in an osmotic microbial fuel cell. J Cleaner Prod. 2017;149:856-62.

21. Borole AP, Mielenz JR, Vishnivetskaya TA, Hamilton CY. Controlling accumulation of fermentation inhibitors in biorefinery recycle water using microbial fuel cells. Biotechnol Biofuels. 2009;2:7.

22. Phung NT, Lee J, Kang KH, Chang IS, Gadd GM, Kim BH. Analysis of microbial diversity in oligotrophic microbial fuel cells using 16S rDNA sequences. FEMS Microbiol Lett. 2004;233(1):77-82.

23. Pham TH, Boon N, De Maeyer K, Hofte M, Rabaey K, Verstraete W. Use of Pseudomonas species producing phenazine-based metabolites in the anodes of microbial fuel cells to improve electricity generation. Appl Microbiol Biotechnol. 2008;80(6):985-93.

24. Park S, Jung YT, Kim S, Yoon JH. Marinobacterium aestuariivivens sp nov., isolated from a tidal flat. Int J Syst Evol Microbiol. 2016;66:1718-23. 
25. Xing F, Xi HB, Yu Y, Zhou YX. A sensitive, wide-ranging comprehensive toxicity indicator based on microbial fuel cell. Sci Total Environ. 2020;703:134667.

26. Fedorovich V, Knighton MC, Pagaling E, Ward FB, Free A, Goryanin I. Novel Electrochemically Active Bacterium Phylogenetically Related to Arcobacter butzleri, Isolated from a Microbial Fuel Cell. Appl Environ Microbiol. 2009;75(23):7326-34.

27. Tan WB, Huang C, Chen C, Liang B, Wang AJ. Bioaugmentation of activated sludge with elemental sulfur producing strain Thiopseudomonas denitrificans X2 against nitrate shock load. Bioresour Technol. 2016;220:647-50.

28. Jiao Y, Zhang GD, Zhao QL. Long-term performance of microbial fuel cell using manure as substrate. Huan jing ke xue. 2014;35(5):1981-7.

29. Si ZH, Song XS, Wang YH, Cao X, Zhao YF, Wang BD, et al. Intensified heterotrophic denitrification in constructed wetlands using four solid carbon sources: Denitrification efficiency and bacterial community structure. Bioresour Technol. 2018;267:416-25.

30. Li JN, Yu YL, Chen DH, Liu GH, Li DY, Lee HS, et al. Hydrophilic graphene aerogel anodes enhance the performance of microbial electrochemical systems. Bioresour Technol. 2020;304:122907.

31. Xia D, Yi XY, Lu Y, Huang WL, Xie YY, Ye H, et al. Dissimilatory iron and sulfate reduction by native microbial communities using lactate and citrate as carbon sources and electron donors. Ecotoxicol Environ Saf. 2019;174:524-31.

32. Tan X, Yang YL, Liu YW, Li X, Fan XY, Zhou ZW, et al. Enhanced simultaneous organics and nutrients removal in tidal flow constructed wetland using activated alumina as substrate treating domestic wastewater. Bioresour Technol. 2019;280:441-6.

33. Rathour R, Patel D, Shaikh S, Desai C. Eco-electrogenic treatment of dyestuff wastewater using constructed wetland-microbial fuel cell system with an evaluation of electrode-enriched microbial community structures. Bioresour Technol. 2019;285:121349.

34. Rago L, Cristiani P, Villa F, Zecchin S, Colombo A, Cavalca L, et al. Influences of dissolved oxygen concentration on biocathodic microbial communities in microbial fuel cells. Bioelectrochem. 2017;116:39-51.

35. Geoffroy V, Payette G, Mauffrey F, Lestin L, Constant P, Villemur R. Strain-level genetic diversity of Methylophaga nitratireducenticrescens confers plasticity to denitrification capacity in a methylotrophic marine denitrifying biofilm. Peerj 2018;6:e4679.

36. Zhang LF, Fu GK, Zhang Z. Electricity generation and microbial community in long-running microbial fuel cell for high-salinity mustard tuber wastewater treatment. Bioelectrochem. 2019;126:20-8.

\section{Figures}



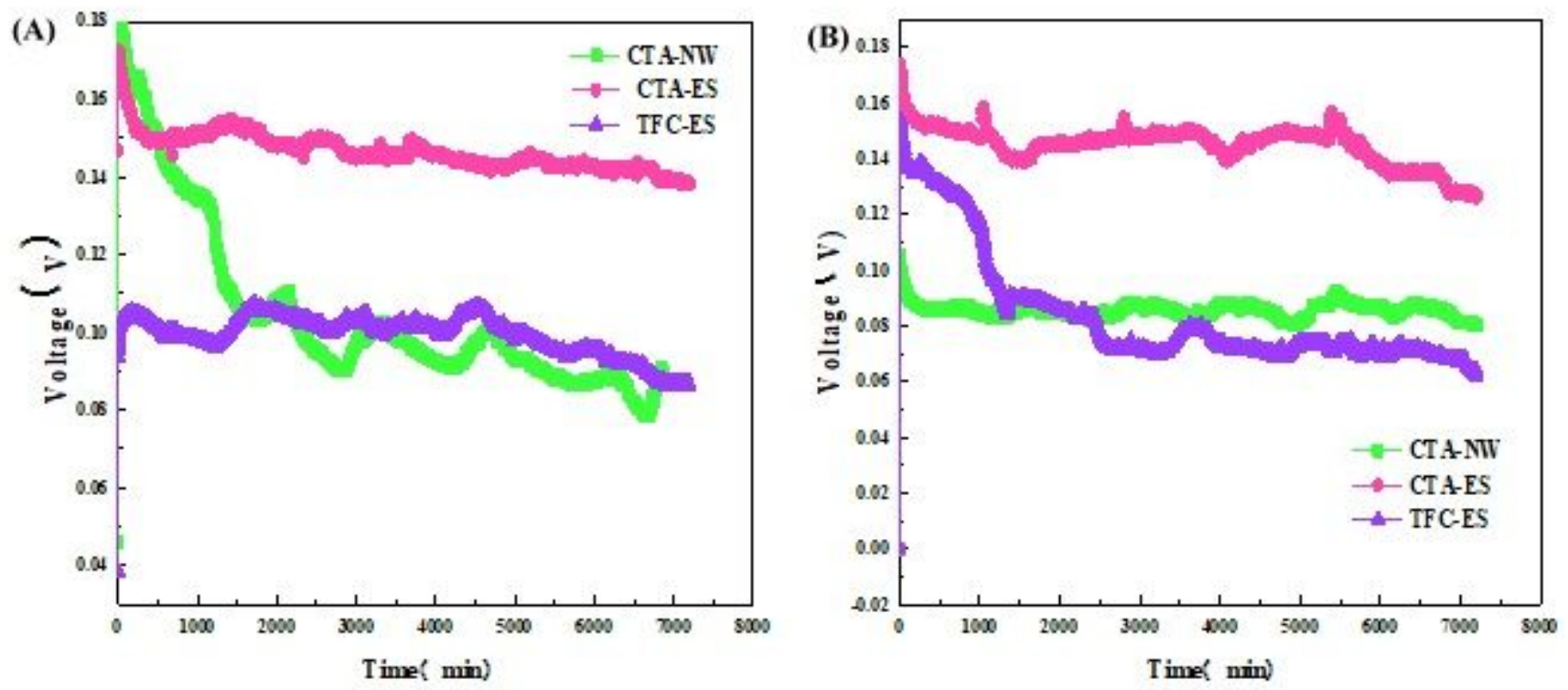

\section{Figure 1}

Voltage outputs versus operation time for OsMFCs fed with leachates under AL-FS mode (A), AL-DS mode (B)
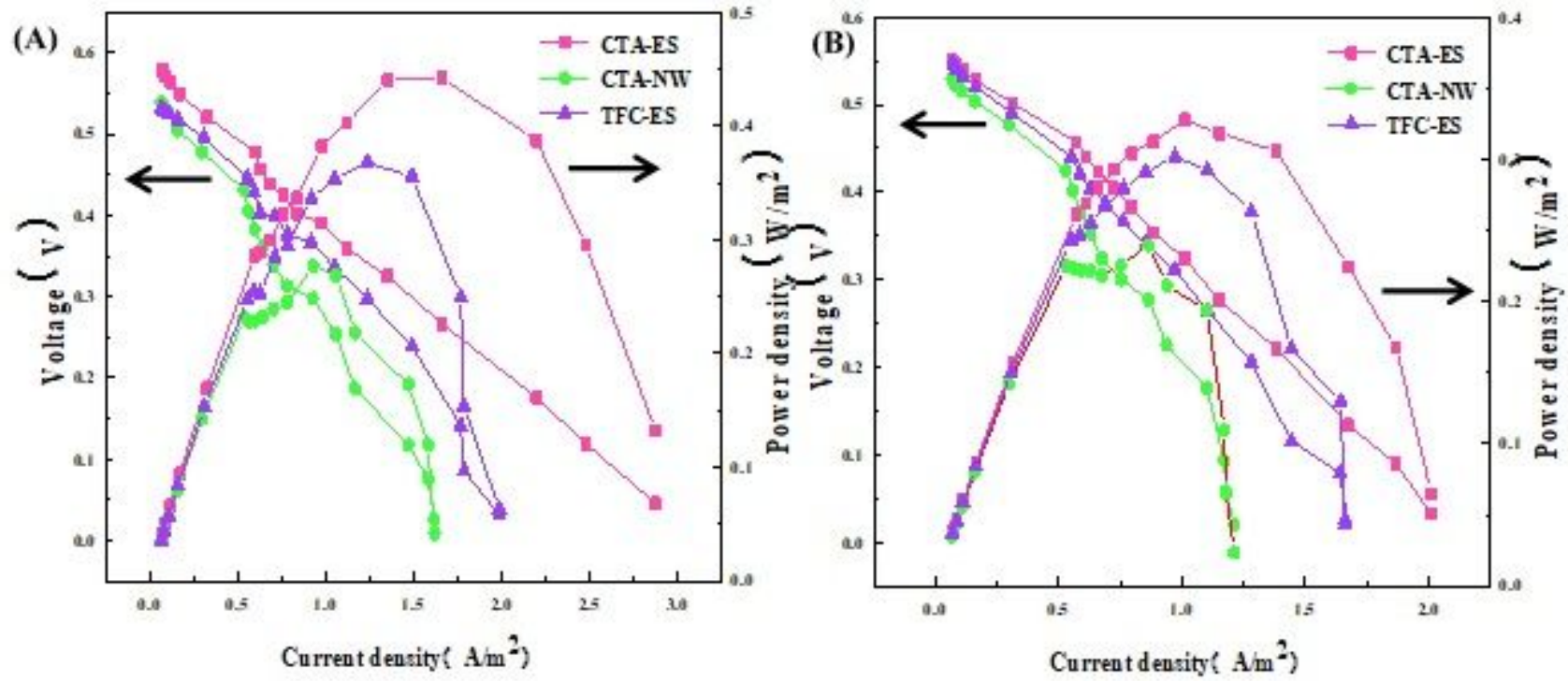

Figure 2

The power density curves and polarization curves of OsMFCs under AL-FSmode (A), AL-DS mode (B) 


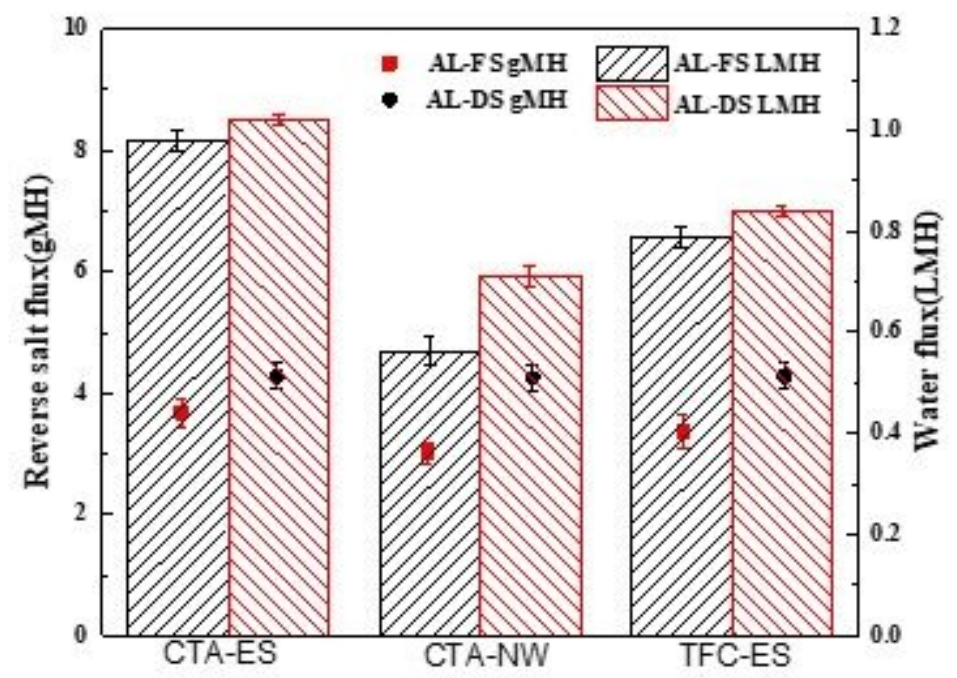

Figure 3

Water flux and reverse salt flux for OsMFCs under both modes
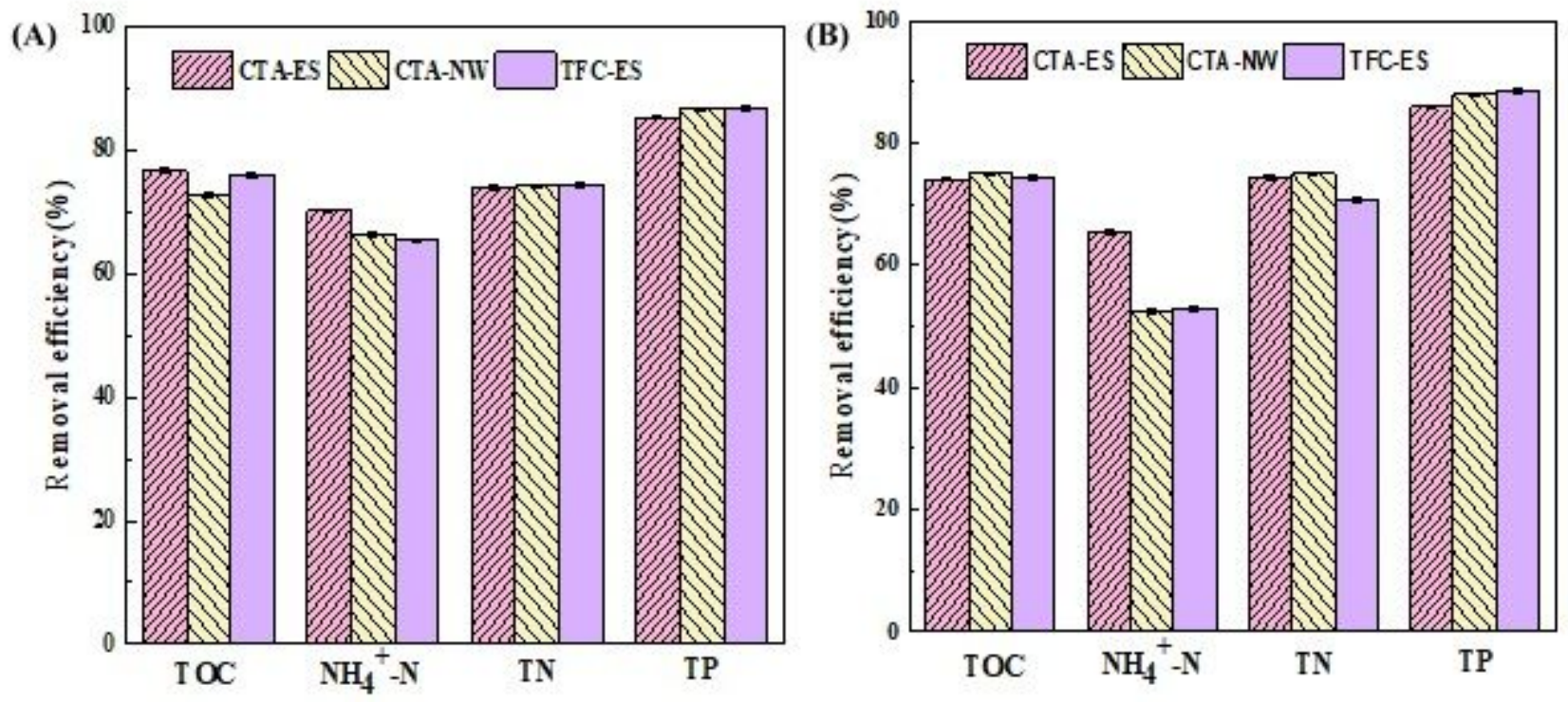

Figure 4

The removal efficiencies after treatment for OsMFC with different FO membrane in AL-FS mode (A), ALDS mode (B) 


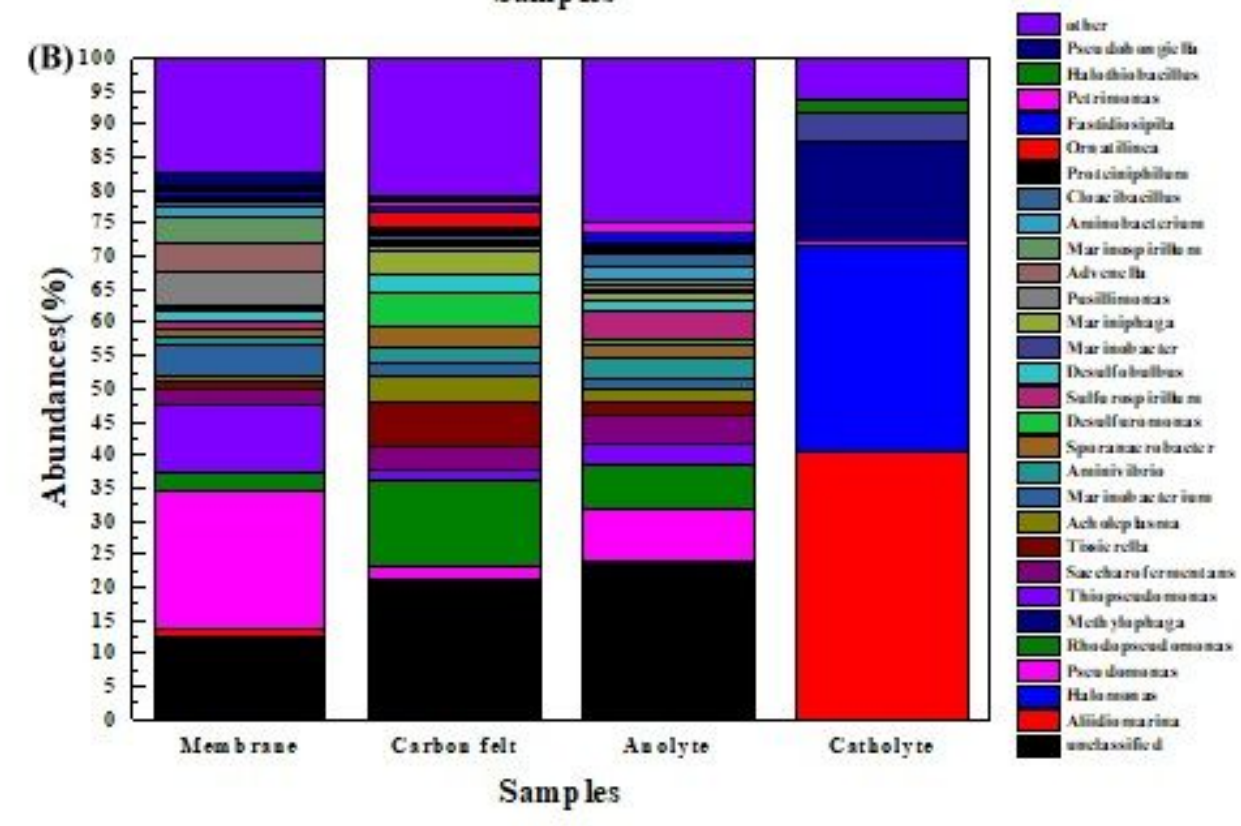

Figure 5

Distribution of microorganisms in each sample of different OsMFCs (A), different site (B) at Genus level

\section{Supplementary Files}

This is a list of supplementary files associated with this preprint. Click to download.

- Supplementaryinformation.pdf 